\title{
A SuperCapacitor Agent for Providing Real-Time Power Services to the Grid
}

\author{
Lorenzo Reyes-Chamorro \\ Mario Paolone \\ Distributed Electrical Systems Laboratory \\ École Polytechnique Fédérale de Lausanne \\ Switzerland \\ \{lorenzo.reyes, mario.paolone\}@epfl.ch
}

\author{
Andrey Bernstein \\ Jean-Yves Le Boudec \\ Laboratory for Communications and Applications 2 \\ École Polytechnique Fédérale de Lausanne \\ Switzerland \\ \{andrey.bernstein, jean-yves.leboudec $\} @$ epfl.ch
}

\begin{abstract}
Supercapacitors-based storage systems are expected to play a key role in microgrids in view of their capability to compensate high-power imbalances. We define an agent for the control of supercapacitor arrays within the context of the novel control framework Commelec, proposed by the Authors as a composable method for real-time control of active distribution networks with explicit power setpoints. An important function of such an agent is to advertise the real-time power capabilities and operational preferences of the supercapacitor array based on local information. Given the small energy capacity of such a device, its internal state can largely vary from one setpoint implementation to the next one. For this reason, the use of an accurate model is crucial in the agent definition. We show that it is possible to infer the real-time power capabilities of the device by using simple measurements on the supercapacitor array suitably coupled with an accurate representation of the cells composing the array. Results show that the agent is able to speak for the resource, thus allowing its use from an external controller.

Index Terms-Supercapacitors, Storage System, Explicit Power Control, Agent-based Control.
\end{abstract}

\section{INTRODUCTION}

The large penetration of renewable energy resources in the distribution level of power systems introduces new complexities for ensuring quality-of-service and reliability of the entire electrical infrastructure due to the stochasticity of these resources. As generation is getting disperse, new technical solutions have emerged. This is the case, for example, of microgrids where generation, storage and controllable demand interact in a low voltage network that can operate both islanded or connected to the main grid as a virtual power plant. The multiplicity of resources with different power flexibility, operation willingness and degrees of uncertainty, added to the fact that they are interdependent through the power network, makes the problem of quality of supply difficult to solve. Note that the elements that play the most important role for the control of the system are the energy storage devices [1]. The classic way for addressing this problem is the droop control method, which has been discussed in the literature extensively [2]-[4]. This solution is based on locally setting the controller parameters on each resource; it can only react to local measurements, and cannot take into account some

\footnotetext{
supported by the SNSF - NRP 70 "Energy Turnaround"
}

global aspects of the system state, such as the state of storage in other resources. Also, it cannot expose the actual internal willingness of the resource and can produce stability issues [5].

It this respect, a composable framework for the real-time control of distribution networks by using explicit power setpoints has been introduced in [6]. In this framework the resources can advertise their current internal needs and power availability by simple messages in order to allow a grid controller to take wise and informed decisions and maintain the state of the system within secure limits. The framework, called Commelec, is designed to be robust (i.e. it avoids the problems inherently posed by software controllers) and scalable (i.e. it easily adapts to grids of any size and complexity). It is based on software agents, which are responsible for resources/subsystems (Resource Agents) or entire grids (Grid Agents) and communicate using a simple, yet powerful protocol with a refresh rate of around $100 \mathrm{~ms}$. See Section II for more details on this framework.

Within this context, in this paper we show how to abstract a supercapacitors-based storage by using the Commelec framework. We provide an accurate mathematical representation of a supercapacitors array that allows for the analytic computation of the power limits and the update of its internal state in realtime. We also show the definition of the associated software agent, whose goal is to respect the internal constraints of the device and expose its flexibility to an external controller. The main challenge is then to accurately abstract the storage capabilities in the time-frame of Commelec. This is achieved by considering a very detailed cell model but simplifying its representation for minimizing the computational effort when computing its real-time capabilities. It is important to note that the proposed problem formulation fits all typical supercapacitors models used in literature. Indeed, several applications of supercapacitors-based storage systems with constant power demand can be found in literature. In particular, applications such as wind power, electrical vehicles and elevators are the typical ones (e.g. [7]-[9]). In general, the power capabilities of the supercapacitors are considered as a linear function of the terminal voltage given by the well-known capacitor's equation. In [10] the sizing of a supercapacitors array is done 
by taking into account the minimum and maximum allowable instantaneous powers using a simplified static model. This approach may not be suitable for following time-varying setpoints with dynamics comparable with the supercapacitors ones while the redistribution phenomenon is taking place [11]. This will require the assessment of the supercapacitors predictive behaviour to feed an optimal controller.

The paper is structured as follows. In Section II we present in detail the requirements defined by the Commelec framework in order to abstract the resource internal state. We present then the design of the agent in Section III. Section

refsec:adv is dedicated to show all the needed information that the agent has to compute. In Section V simulation results using a detailed model are presented. Finally Section VI outlines the conclusions of the work.

\section{The Commelec Framework}

\section{A. Agents Advertisements}

In the Commelec framework, a software agent is associated with a resource (henceforth called "Resource Agent", RA), or an entire system including a grid and/or a number of devices (henceforth called "Grid Agent", GA). There is a welldefined relationship between the agents, which follows from the tree structure of the distribution networks. An example of agents relationship is shown in Figure 1 where GA is in charge of controlling RAs $A_{1}, \ldots, A_{N}$, who are responsible for subsystems $S_{1}, \ldots, S_{N}$.

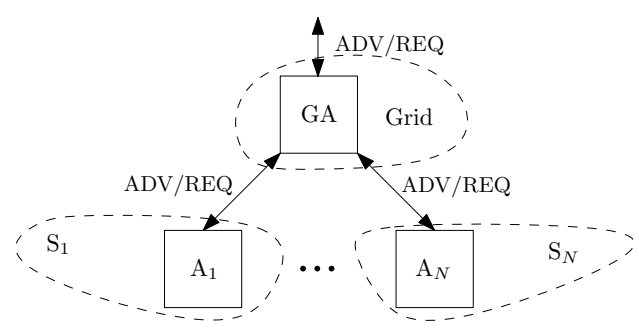

Fig. 1. A general scheme for showing agents interaction.

Each resource agent advertises its internal state to its grid agent using the following three elements. (1) The $P Q$ profile $\mathcal{A}$ is the region in the $(P, Q)$-plane (for active and reactive power) that the subsystem under the control of this resource agent can deploy. (2) The virtual cost $C$ is a function, defined for every $(P, Q)$ in the $P Q$ profile, which returns a number $C(P, Q)$ interpreted as the willingness of this subsystem to apply a requested power setpoint $(P, Q)$. (3) The belief function $B F$ returns the set of all possible (actual) setpoints that this subsytem might in reality implement when instructed to implement a target setpoint. It accounts for the uncertainty of the subsystem operation. For subsystems such as PV/wind farms, or loads, the belief function returns larger sets, to account for their volatility.

It is important to observe that these three elements (i) are the only information needed for real-time control in the Commelec framework and (ii) abstract the specific internal state of the resource.

\section{B. Operation of a Resource Agent}

For any resource agent in the Commelec framework, the sending/receiving message cycle is repeated continuously and endlessly in normal operation. In this sense, a RA computes an advertisement just after receiving a request. The whole cycle of a RA is described next (see Fig. 2).

1) Reception of a new setpoint request $(P, Q)_{t_{0} \rightarrow t_{1}}$ at $t_{0}$. The request is to maintain this power fixed from $t_{0}$ to $t_{1}=t_{0}+\Delta t . \Delta t$ is defined by the GA.

2) Check the feasibility of the requested setpoint given the actual instantaneous measured/estimated state in the resource (not available to the GA). Note that for resources with high uncertainty, the implemented power may significantly differ from the requested setpoint, while it will be exactly the same (ideally) for fully controllable resources. This uncertainty has been advertised in the previous belief function. This action also serves to maintain the resource safe in case the GA requests an invalid power setpoint.

$3)$ Instruct the resource to implement the verified setpoint $(\tilde{P}, \tilde{Q})_{t_{0} \rightarrow t_{1}}$.

4) Compute and send the advertisement $(\mathcal{A}, B F, C)_{t_{0}}$ based on local measurements. This advertisement should ensure that $(P, Q)_{t_{1} \rightarrow t_{2}}$ maintains the resource safe until $t_{2}$.

5) Wait for a new request from GA.

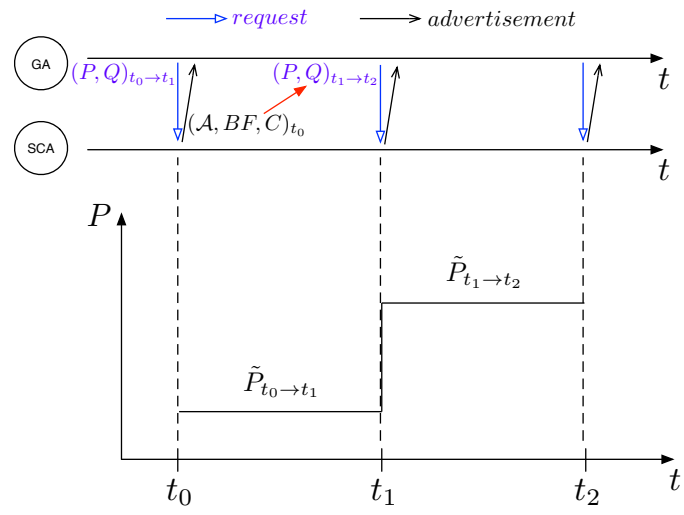

Fig. 2. Representation of the messages between GA and RA in time. The red arrow indicates that $(P, Q)_{t_{1} \rightarrow t_{2}}$ is strongly dependant on $(\mathcal{A}, B F, C) t_{0}$. The lower diagram shows the implementation of the requested setpoint in time. For simplicity only active power is shown.

\section{Design of SuperCapacitor Agent (SCA)}

In this section we present the design of a SuperCapacitor Agent (SCA), which is a resource agent associated with a supercapacitors array.

\section{A. Specificity of the SCA}

For storage systems characterized by relatively high-energy densities (e.g., batteries), the update rate of the resource setpoint imposed by the Commelec framework (around 100ms) is small enough so that the state of charge can normally be considered constant between two consecutive setpoints. In contrast, for a supercapacitor array, this assumption is no longer 
true. Unlike battery energy storage systems, supercapacitors are characterized by a higher power density and a smaller energy density [12]. Therefore, operation capabilities of a supercapacitors array for the next time step will depend on the last implemented power setpoints.

As a result, the SCA must have an accurate model in order to assess the electrical state of its resource. For this purpose, we rely on the supercapacitor cell model presented in [13], where the redistribution of residual charge phenomena is taken into account. A brief description of the model is given in the next section in order to provide the elements needed for the SCA definition.

As the Commelec framework controls AC power flows, the SCA needs to consider the presence of a power converter. A generic model of a power converter is presented in [14]. It is composed by an algebraic transfer function that represents the losses taking place in the device. In view of this assumption, a setpoint $(P, Q)$ on the $\mathrm{AC}$ side of the converter is transformed into a setpoint $p$ on the DC side of the converter and, therefore, on the supercapacitors array. The mapping between a $(P, Q)$ setpoint and a $p$-setpoint is presented in [14] for resources with $\mathrm{AC} / \mathrm{DC}$ converters and it is no further discussed here.

We focus now in the computation of the limitations of the storage device on the DC side assuming a constant power request between two subsequent deployed setpoints (see Sections III-C and III-D). More precisely, the SCA needs to compute the set of DC power setpoints that can be safely implemented.

\section{B. Supercapacitor cell model}

Fig. 3 shows the model of a supercapacitor cell [13].

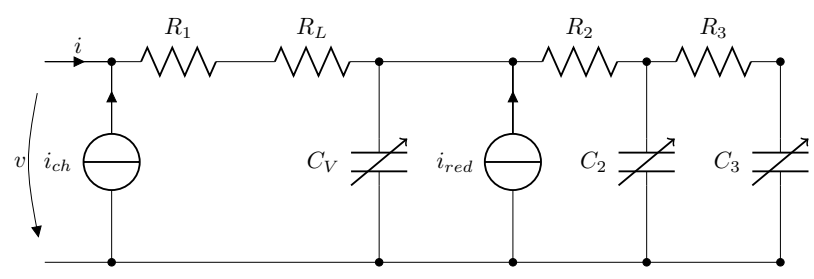

Fig. 3. Adopted supercapacitor model.

The peculiarity of this model is that it is capable to represent the so-called redistribution of the residual charge taking place in each cell of the array. The residual charge $\left(Q_{R}\right)$ is the amount of charge that remains stored into the SC after a discharge that resulted into a SC terminals voltage $(v)$ achieved at the end of the discharge [13]. The modeling of $Q_{R}$ redistribution is accounted by two current sources $i_{c h}$ and $i_{\text {red }}$ in the supercapacitor equivalent circuit (Fig. 3). Note that $i_{c h}$ is only active when the SC is in operation, namely $i>0$ (charging) or $i<0$ (discharging), while $i_{\text {red }}$ is inactive. On the contrary, $i_{\text {red }}$ is active when $i=0$ and $i_{c h}$ is inactive. The supercapacitor cell can then be represented as a system of 6 state variables $\mathbf{x}=\left(v_{C_{V}}, v_{C_{2}}, v_{C_{3}}, i_{c h}, i_{\text {red }}, i_{\text {red }}\right)$, where $v_{C_{V}}, v_{C_{2}}$ and $v_{C_{3}}$ are the internal capacitors voltages and, $i_{c h}$ and $i_{\text {red }}$ the current magnitude of the new current sources.
The system can be defined as a linear control ODE problem where $\mathbf{x}$ is the state, $i$ the input and $v$ the output ${ }^{1}$.

$$
\begin{gathered}
\dot{\mathbf{x}}=\mathbf{A} \mathbf{x}+\mathbf{B} i \\
v=\mathbf{C} \mathbf{x}+D i \\
\mathbf{x}\left(t_{1}\right)=\mathbf{x}_{1}
\end{gathered}
$$

For the implementation of the SCA, we consider the following assumptions:

- There is access to the instantaneous measurement of $v$ and $i$. With this measurement available, and given initial conditions, we can compute the state of the model at any time step.

- The parameters of the model are known by means of the process described in [13].

- The SC array is made of identical cells connected in series so that we can directly aggregate them. Therefore, the status of a cell inherently identifies the one of the entire array $^{2}$.

\section{The SCA Problem}

The SC array has operational limits in terms of voltage and current magnitudes: $v_{\max }>0, v_{\min }>0, i_{\max }>0$ and $i_{\min }<0$. In practice the current limits will be mostly defined by the dc-dc stage of the power converter interface.

Observation III.1. For the cell model presented in [13], both the voltage and the current are monotonic with respect to the power. In Fig. 4, we present a numerical validation of this property applied to the cited model. Each curve represents the evolution of $v$ and $i$ with respect to $p$ for different initial conditions. The power-range of each curve is defined by the operational limits of the cell.
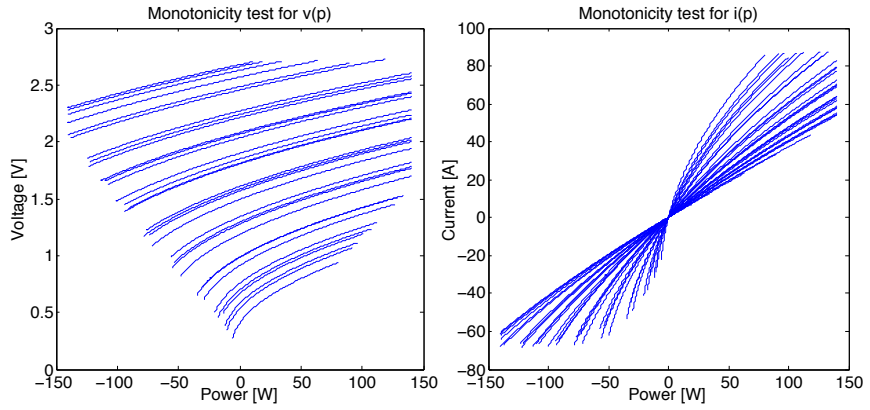

Fig. 4. Results of the computation of $v(p)$ and $i(p)$ for different initial conditions. The values are computed for a single cell of [13].

The SCA problem is to compute, at time $t_{0}$, the set of all DC-side power setpoints $p=p_{t_{1} \rightarrow t_{2}}$ that are feasible, i.e. for which $v$ and $i$ are inside their operational constraints during the whole interval $\left[t_{1}, t_{2}\right]$. It follows that, given Obs. III.1, the set of feasible DC-power setpoints is an interval, and it is

\footnotetext{
${ }^{1}$ The details on the equivalence of (1) and (2) with the supercapacitor model are discussed in the Appendix.

${ }^{2}$ Note that the distribution of charge among cells is out of the scope of the used model.
} 
sufficient to compute the maximum and minimum DC-powers $p_{\min }$ and $p_{\max }$. The SCA problem at $t_{0}$ is therefore:

minimize (resp. maximize) $\quad p_{t_{1} \rightarrow t_{2}}$ subject to

$$
\begin{aligned}
& p(t)=v(t) i(t) \\
& v_{\min } \leq v(t) \leq v_{\max } \\
& i_{\min } \leq i(t) \leq i_{\max } \\
& \text { Eqs. }(1),(2) \text { and }(3) \\
& \text { for } t \in\left[t_{1}, t_{2}\right]
\end{aligned}
$$

\section{Solution of the SCA Problem}

First recall that the power $p$ is kept fixed for the considered time-window. We can develop Eq. (2) to compute $v$ as a function of $p$, when $i \neq 0$ :

$$
\begin{array}{r}
v=\mathbf{C} \mathbf{x}+D \frac{p}{v} \\
v^{2}-\mathbf{C} \mathbf{x} v-D p=0 \\
v=\frac{\mathbf{C} \mathbf{x} \pm \sqrt{(\mathbf{C} \mathbf{x})^{2}+4 D p}}{2} .
\end{array}
$$

Assuming that $v$ is always between the bounds, it is always positive. Then, the only valid solution of Eq. (4) when $p>0$ is the one linked to the positive sign, since $D>0$ (see the Appendix). Note that the argument of the square root is negative for $p<p_{c}=-\frac{\left(\mathbf{C x}\left(t_{2}\right)\right)^{2}}{4 D}$. Therefore, the correct operation of the supercapacitors array requires $p \geq p_{c}+\delta$, where $\delta$ is a safety margin suitably defined. The physical meaning of this power is the following: for values lower than $p_{c}$, the electrical state of the array is so deteriorated that no actual power can be provided by the device. As a consequence the solution with the negative sign in Eq. (4) can be never reached by continuity argument, i.e., we always use the positive sign also when $p<0$. Applying this to Eq. (1) the non-linear ODE problem to be solved is:

$$
\begin{aligned}
\dot{\mathbf{x}} & =\mathbf{A} \mathbf{x}+\mathbf{B} i \\
& =\mathbf{A} \mathbf{x}+\frac{\mathbf{B}}{D}(v-\mathbf{C} \mathbf{x}) \\
& =\left(\mathbf{A}-\frac{\mathbf{B} \mathbf{C}}{D}\right) \mathbf{x}+\frac{\mathbf{B}}{D} v \\
& =\left(\mathbf{A}-\frac{\mathbf{B C}}{D}\right) \mathbf{x}+\frac{\mathbf{B}}{D}\left(\frac{\mathbf{C x}+\sqrt{(\mathbf{C x})^{2}+4 D p}}{2}\right) \\
\dot{\mathbf{x}} & =\left(\mathbf{A}-\frac{\mathbf{B C}}{2 D}\right) \mathbf{x}+\frac{\mathbf{B}}{2 D} \sqrt{(\mathbf{C x})^{2}+4 D p},
\end{aligned}
$$

which solution is only obtained through numerical methods assuming a given value on the input $p$.

When $p=0$, then $i=0$ and we can directly solve Eq. (1):

$$
\begin{array}{r}
\mathbf{x}=e^{\mathbf{A} t} \mathbf{x}_{\mathbf{0}} \\
v=\mathbf{C} e^{\mathbf{A} t} \mathbf{x}_{\mathbf{0}}
\end{array}
$$

The computation of the initial conditions $\mathbf{x}_{\mathbf{0}}$ for both cases ( $i=0$ and $i \neq 0$ ) is presented in the Appendix.

We now present how to compute the DC power bounds for the period $t_{1} \rightarrow t_{2}$ at time $t_{0}$. Recall that from the viewpoint of the resource, $p>0$ represents charging and $p<0$ discharging.
1) At $t_{0}$ the implemented constant power from $t_{0} \rightarrow t_{1}$ is known. With this, compute the state $\mathbf{x}\left(t_{1}\right)$ by solving Eq. (5) with $p=p_{t_{0} \rightarrow t_{1}}$.

2) Use (6) in the window $\left[t_{1}, t_{2}\right]$, with the purpose of verifying that the redistribution of charges does not violate the voltage limits. Store the value $v_{\text {test }}=\left.v\left(t_{2}\right)\right|_{p=0}{ }^{3}$.

3) Computation of $p_{\max }$ : first, note that in a charging phase when $p>0, v$ always increases (see Obs. III.1). As $p$ is constant during this phase, the maximum magnitude of $i$ is reached exactly at the beginning of the new operation window. Hence, the corresponding power that will attain this condition is given by $p_{\max }^{i}=\mathbf{C x}\left(t_{1}\right) i_{\max }+D i_{\max }^{2}$. Then, by performing a binary search, we look for the value of $p$ that gives $v\left(t_{2}\right)=v_{\max }$. This power is named $p_{\text {max }}^{v}$. If $v_{\text {test }}>v_{\max }$, we perform the search in the range $\left[v_{\max } i_{\min }, 0\right]$, otherwise in $\left[0, v_{\max } i_{\max }\right]$.

We finally compute $p_{\max }=\min \left(p_{\max }^{i}, p_{\max }^{v}\right)$.

4) Computation of $p_{\min }$ : as for $p_{\max }$, we perform a binary search for the value of $p$ such that it reaches $i=\max \left(i_{\text {min }}, \frac{v_{\text {min }}-\mathbf{C x}\left(t_{2}\right)}{D}\right)$. If $v_{\text {test }}<v_{\text {min }}$ the search is done in the range $\left[0, v_{\max } i_{\max }\right]$, otherwise in $\left[\max \left(p_{c}, v_{\max } i_{\min }\right), 0\right]$.

\section{SCA ADVERTISEMENT}

As mentioned in section II the advertisement sent by the RA is composed by three elements: $P Q$ profile $\mathcal{A}$, belief function $B F$ and virtual cost $C$.

\section{A. $P Q$ profile}

The PQ power capabilities of the supercapacitors array are defined by the previously computed limits, suitably transferred to the AC terminal through the power converter. The power limits on the AC side are called $P_{\min }$ and $P_{\max }{ }^{4}$. As in [14] we model the converter as a static element with fixed efficiency. The intersection between these limits and the power converter capabilities -which is considered as the disk in the PQ plane with center in the origin and radius its rated powerwill define the $P Q$ profile (for simplicity only the P-coordinate is presented).

$$
\mathcal{A}(P)=\left[P_{\text {min }}, P_{\text {max }}\right] \cap\left[P_{\text {min }}^{C}, P_{\text {max }}^{C}\right]
$$

\section{B. Belief function}

Considering that the supercapacitors array is connected through a power converter to the grid, we assume that it is fully controllable, namely, it can follow exactly the requested power. This means that we can define $B F(P, Q)=\{(P, Q)\}$, where the singleton $\{(P, Q)\}$ is the power request.

\section{Virtual cost}

We define $C$, as a function of the current state-of-charge $(S o C)$ and therefore only depending on the active power $P$. We assume that there is a desirable or target value $\left(S o C_{T}\right)$

\footnotetext{
${ }^{3}$ For example, if the redistribution phase causes the voltage to increase and violate $v_{\max }, p_{\max }$ will be negative (i.e., the array should be discharged).

${ }^{4}$ Note that in the AC side, $P>0$ is for discharging.
} 
defined externally (e.g. system-wise with a long-term optimizer). Therefore, we set a higher cost for powers that make $\Delta S o C=S o C-S o C_{T}$ larger. A suitable quadratic function is desirable for providing convexity.

We consider that the $S o C$ is computed as the ratio between the charge stored in all the capacitors of the model $\left(C_{V}\right.$, $C_{2}$ and $\left.C_{3}\right)$ and the nominal capacity $Q_{n}{ }^{5}=\left(C_{V}+C_{2}+\right.$ $\left.C_{3}\right)\left(v_{\max }-v_{\min }\right)$ :

$$
\begin{gathered}
S o C=\frac{1}{Q_{n}}\left(C_{V} v_{C_{V}}+C_{2} v_{C_{2}}+C_{3} v_{C_{3}}+Q_{R}\right) \\
Q_{R}= \begin{cases}\Delta Q_{1} & \text { if } i \neq 0 \\
\Delta Q_{3} & \text { if } i=0^{6}\end{cases}
\end{gathered}
$$

Hence, the cost function that we have adopted is:

$$
\begin{gathered}
C(P, Q)=\frac{\Delta S o C}{3}\left(\operatorname{sgn}(\Delta S o C) P^{2}-2 P\right) . \\
\text { V. SimUlation RESUlts }
\end{gathered}
$$

In this section we show the application of our method to a supercapacitors-based energy storage device of 120kVA and $300 \mathrm{Wh}$ (equivalent to 853 cells of [13] in series).

In Fig. 5 we present the results of a 50s simulation where different power setpoints ("Request") are provided by an external controller (the GA in the Commelec framework). The DC current limit of the converter is set to $80 \mathrm{~A}$. We show the evolution of $v, i$, the AC power, the power limits and the state-of-charge.

The system starts in a resting phase for $1 \mathrm{~s}(p=0)$ with a $S o C=0.5 p u$. At $2 \mathrm{~s}$ the GA requests to charge at full power (120kVA) but soon (around 7s) the SCA advertises to the GA a power limitation, thus avoiding the violation of $v_{\max }$, the charge continues reducing the current magnitude while maintaining $v=v_{\max }$. This can be also seen in the $S o C$ plot, as the supercapacitors array gets almost full in this phase. At $12 \mathrm{~s}$ the request is null, and the array goes into a redistribution phase. The voltage decreases slowly due to the redistribution of charges in the cells. At 22s a discharging phase with constant power (inside the limits) causes the voltage to decrease and then the current to increase. The current limitation is soon reached (around 25s) and the power magnitude decreases accordingly. At around $28 \mathrm{~s}$ the voltage magnitude is so deteriorated that the $p_{c}$ limit is reached and the current magnitude is reduced. At $32 \mathrm{~s}$ a new charge starts but as the voltage magnitude is so low, the $i_{\max }$ limit is rapidly activated. Since the voltage starts increasing, both $P_{\min }$ and $P_{\max }$ increase in magnitude and the system is charged at constant power from second 34. At 40s the GA requests again the injection of power into the grid and the voltage starts decreasing while the power is limited first by $i_{\min }$ and then by $p_{c}$.

It is interesting to see the rapid evolution of the $S o C$ during short-time windows. As a function of this rapid $S o C$

\footnotetext{
${ }^{5} Q_{n}$ represents the effective charge that the supercapacitors array can store since it cannot be operated until $v=0$ as the DC voltage is bounded by the power electronics interface.
}

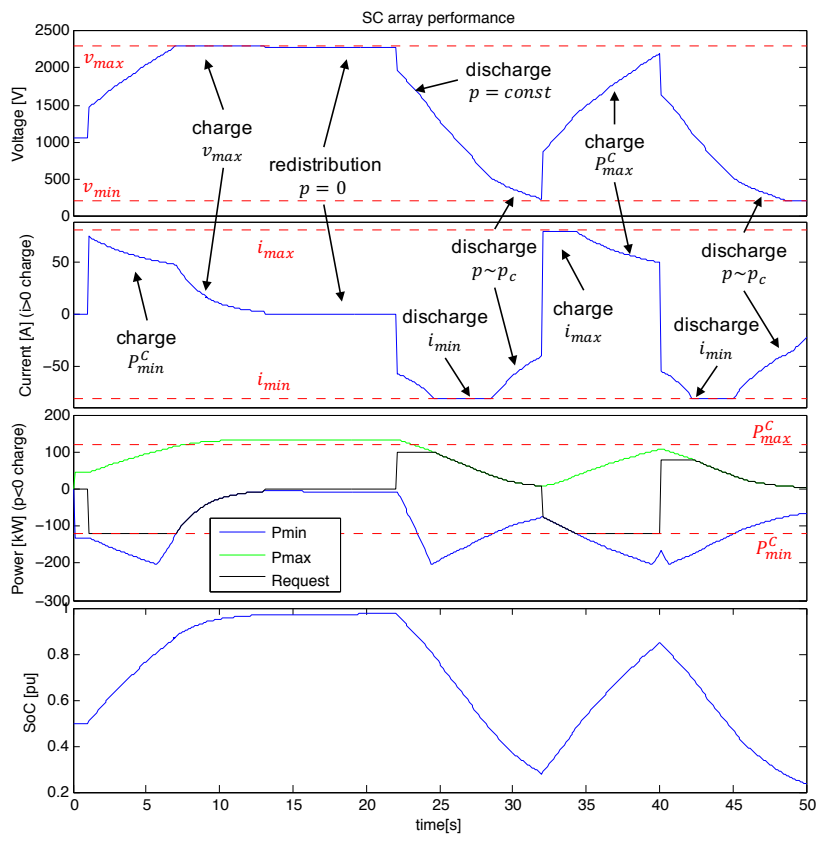

Fig. 5. Simulation results. The red dashed lines represent the operation limits for each variable. In the case of the power plot, it represents the power converter capabilities (120kVA). The actual $P Q$ profile (in the P-coordinate) is $\mathcal{A}(P)=\left[P_{\min }, P_{\max }\right] \cap\left[P_{\text {min }}^{C}, P_{\text {max }}^{C}\right]$.

dynamic, the proposed process is rapidly capable to compute and advertise the internal state of the supercapacitors array through the Commelec framework.

\section{CONCLUSION}

We have proposed a method to expose the power operation limits of a supercapacitors-based energy storage system by maintaining both voltage and current magnitudes always inside feasible values. The method also allows to follow explicit power setpoints sent from external controllers in order to provide power services to the grid. We have shown that given the rapid dynamic of this kind of storage system, the implementation of such a method is necessary for allowing real-time control. More specifically, the proposed method, allows for the definition of a supercapacitors array agent within the Commelec control framework.

\section{APPENDIX}

\section{Model equivalence}

We present in this section how to derive equations (1) and (2) according to the SC model [13]. As previously mentioned, the current sources in the model of Fig. 3 are active or inactive depending upon the current magnitude $i$. Indeed, when $i=0$ only $i_{\text {red }}$ is active and when $i \neq 0$ only $i_{c h}$ is. We differentiate this two cases as redistribution mode and charging/discharging mode respectively. In general $\mathbf{A}$ can be expressed as:

$$
\mathbf{A}=\left(\begin{array}{lll}
\mathbf{A}_{11} & \multicolumn{2}{c}{\mathbf{A}_{12}} \\
& A_{c h} & \mathbf{0}_{1 \times 2} \\
\mathbf{0}_{3 \times 3} & \mathbf{0}_{2 \times 1} & \mathbf{A}_{\text {red }}
\end{array}\right)
$$


For both cases $\mathbf{A}_{11}$ can be defined as:

$$
\mathbf{A}_{11}=\left(\begin{array}{ccc}
\frac{-1}{C_{V} R_{2}} & \frac{1}{C_{V} R_{2}} & 0 \\
\frac{1}{C_{2} R_{2}} & -\left(\frac{1}{C_{2} R_{2}}+\frac{1}{C_{2} R_{3}}\right) & \frac{1}{C_{2} R_{3}} \\
0 & \frac{1}{C_{3} R_{3}} & -\frac{1}{C_{3} R_{3}}
\end{array}\right)
$$

For the redistribution mode $(i=0)$ :

$$
\begin{gathered}
\mathbf{A}_{12}=\left(\begin{array}{ccc}
0 & \frac{1}{C_{V}} & 0 \\
0 & 0 & 0 \\
0 & 0 & 0
\end{array}\right), \mathbf{A}_{\text {red }}=\left(\begin{array}{cc}
0 & 1 \\
-\frac{1}{\tau_{2} \tau_{3}} & -\left(\frac{1}{\tau_{2}}+\frac{1}{\tau_{3}}\right)
\end{array}\right) \\
\mathbf{C}=\left(\begin{array}{llllll}
1 & 0 & 0 & 0 & 0 & 0
\end{array}\right)
\end{gathered}
$$

$A_{c h}, \mathbf{B}$ and $D$ can be defined as zero.

For the charging/discharging mode $(i \neq 0)$ :

$$
\begin{gathered}
\mathbf{A}_{12}=\left(\begin{array}{ccc}
\frac{1}{C_{V}} & 0 & 0 \\
0 & 0 & 0 \\
0 & 0 & 0
\end{array}\right), \mathbf{B}^{T}=\left(\begin{array}{cccccc}
\frac{1}{C_{V}} & 0 & 0 & 0 & 0 & 0
\end{array}\right) \\
A_{c h}=-\frac{1}{\tau}, \quad \mathbf{C}=\left(\begin{array}{llllll}
1 & 0 & 0 & R_{1}+R_{L} & 0 & 0
\end{array}\right) \\
D=R_{1}+R_{L}
\end{gathered}
$$

$\mathbf{A}_{\text {red }}$ can be defined as zero. The parameters $\tau, \tau_{1}$ and $\tau_{2}$ are the time constants of the two current sources.

\section{Initial conditions}

Given the duality of the problem, new initial conditions for the state variables $i_{c h}, i_{\text {red }}$ and $i_{\text {red }}$ have to be computed on each mode transition. In [13] the details for computing these values are discussed. Here we present the results useful for our formulation.

For the transition to the redistribution mode:

$$
i_{\text {red }}(0)=\left(\frac{\eta_{2}}{\tau_{2}}+\frac{\eta_{3}}{\tau_{3}}\right) \Delta Q_{4}, i_{\text {red }}(0)=-\left(\frac{\eta_{2}}{\tau_{2}^{2}}+\frac{\eta_{3}}{\tau_{3}^{2}}\right) \Delta Q_{4},
$$

where $\eta_{2}$ and $\eta_{3}$ are parameters of the model and $\Delta Q_{4}$ is the quota of charge contributing to the redistribution phase due to the diffusion phenomena. This is the effective charge that will be redistributed in the cell during this phase. It is important to note that this charge is the result of the difference between the available charge for redistribution at the beginning of the last charging/discharging phase $(\Delta Q)$ and the charge that was indeed redistributed $\left(\Delta Q_{1}\right)$ and the amount of residual charge that the cell would have in case it stays in open-circuit conditions $\left(\Delta Q_{2}\right)$, i.e., $\Delta Q_{4}=\Delta Q-\Delta Q_{1}-\Delta Q_{2}$. We define as $\Delta Q_{3}$ the charge is effectively redistributed during this phase, i.e., the integral of $i_{\text {red }}$ :

$$
\Delta Q_{3}=\eta_{2} \Delta Q_{4}\left(1-e^{-\frac{t}{\tau_{2}}}\right)+\eta_{3} \Delta Q_{4}\left(1-e^{-\frac{t}{\tau_{3}}}\right) .
$$

For the transition to the charging/discharging mode:

$$
i_{c h}(0)=\frac{\Delta Q}{\tau},
$$

where $\Delta Q=\Delta Q_{4}-\Delta Q_{3}+\Delta Q_{2}$. As mentioned before, $\Delta Q_{1}$ is the charge that was redistributed during the charging/discharging mode, i.e., the integral of $i_{c h}$ :

$$
\Delta Q_{1}=\Delta Q\left(1-e^{-\frac{t}{\tau}}\right) .
$$

The initial conditions for all other state variables are simply taken as the state in the previous step.

\section{REFERENCES}

[1] K.C. Divya and J. Ostergaard. Battery energy storage technology for power systems - an overview. Electric Power Systems Research, 79:511520, 2009.

[2] J. A. Peças Lopes, C. L. Moreira, and A. G. Madureira. Defining control strategies for microgrids islanded operation. IEEE Transactions on Power Systems, 21(2):916-924, 2006.

[3] F. Katiraei and M. R. Iravani. Power management strategies for a microgrid with multiple distributed generation units. IEEE Transactions on Power Systems, 21(4):1821-1831, Nov. 2006.

[4] José Matas, Miguel Castilla, Luis García de Vicuña, Jaume Miret, and Juan Carlos Vásquez. Virtual impedance loop for droop-controlled single-phase parallel inverters using a second-order general-integrator scheme. IEEE Transactions on Power Electronics, 25(12):2993 - 3002, December 2010.

[5] D.E. Olivares, A. Mehrizi-Sani, A.H. Etemadi, C.A. Cañizares, R. Iravani, M. Kazerani, A. H. Hajimiragha, O. Gomis-Bellmunt, M. Saeedifard, R. Palma-Behnke, G.A. Jimenez-Estevez, and N.D. Hatziargyriou. Trends in microgrid control. IEEE Transactions on Smart Grid, 5(4):1905-1919, 2014.

[6] A. Bernstein, L. Reyes-Chamorro, J.-Y. Le Boudec, and M. Paolone. A composable method for real-time control of active distribution networks with explicit power setpoints. Part i: Framework. Electric Power Systems Research, 125:254-264, 2015.

[7] A. Rufer and P. Barrade. A supercapacitor-based energy-storage system for elevators with soft commutated interface. IEEE Transactions on Industry Applications, 38(5):1151-1159, 2002.

[8] N. Mendis, K. M. Muttaqi, and S. Perera. Management of batterysupercapacitor hybrid energy storage and synchronous condenser for isolated operation of pmsg based variable-speed wind turbine generating systems. IEEE Transactions on Smart Grid, 5(2):944-953, 2014.

[9] R. E. Araújo, R. de Castro, C. Pinto, P.Melo, and D. Freitas. Combined sizing and energy management in evs with batteries and supercapacitors. IEEE Transactions on Vehicular Technology, 63(7):3062-3076, 2014.

[10] A. Kuperman, M. Mellincovsky, C. Lerman, I. Aharon, N. Reichbach, G. Geula, and R. Nakash. Supercapacitor sizing based on desired power and energy performance. IEEE Transactions on Power Electronics, 29(10):5399-5405, 2014.

[11] M. Kaus, J. Kowal, and D. U. Sauer. Modelling the effects of charge redistribution during self-discharge of supercapacitors. Electrochimica Acta, 55(25):7516-7523, 2010.

[12] T.A. Smith, J.P. Mars, and G.A. Turner. Using supercapacitors to improve battery performance. In Proceedings of the 2002 IEEE 33rd Annual Power Electronics Specialists Conference, volume 1, pages 124128, 2002.

[13] D. Torregrossa, M. Bahramipanah, E. Namor, R. Cherkaoui, and M. Paolone. Improvement of dynamic modeling of supercapacitor by residual charge effect estimation. IEEE Transactions on Industrial Electronics, 61(3):1345 - 1354, April 2013.

[14] L. Reyes-Chamorro, A. Bernstein, J.-Y. Le Boudec, and M. Paolone. A composable method for real-time control of active distribution networks with explicit power setpoints. Part ii: Implementation and validation. Electric Power Systems Research, 125:265-280, 2015. 